\title{
Reflectance-difference spectroscopy study of the Fermi-level position of low-temperature-grown GaAs
}

\author{
Y. H. Chen, ${ }^{*}$ Z. Yang, ${ }^{\dagger}$ R. G. Li, ${ }^{\ddagger}$ and Y. Q. Wang \\ Department of Physics, Hong Kong University of Science and Technology, Clearwater Bay, Kowloon, Hong Kong \\ Z. G. Wang \\ Laboratory of Semiconductor Materials Science, Institute of Semiconductors, Chinese Academy of Sciences, \\ Box 912, Beijing 100083, China \\ (Received 11 November 1996)
}

\begin{abstract}
The results of a reflectance-difference spectroscopy study of GaAs grown on (100) GaAs substrates by low-temperature molecular-beam epitaxy (LT-GaAs) are presented. In-plane optical anisotropy resonances which come from the linear electro-optic effect produced by the surface electric field are observed. The RDS line shape of the resonances clearly shows that the depletion region of LT-GaAs is indeed extremely narrow $(\ll 200 \AA)$. The surface potential is obtained from the RDS resonance amplitude without the knowledge of space-charge density. The change of the surface potential with post-growth annealing temperatures reflects a complicated movement of the Fermi level in LT-GaAs. The Fermi level still moves for samples annealed at above $600{ }^{\circ} \mathrm{C}$, instead of being pinned to the As precipitates. This behavior can be explained by the dynamic properties of defects in the annealing process. [S0163-1829(97)51712-7]
\end{abstract}

GaAs grown by low-temperature molecular-beam epitaxy (LT-GaAs) has recently attracted much interest due to its unique properties and potential electronic and optoelectronic applications. ${ }^{1-5}$ This material is crystalline, with a high content of excess arsenic, ${ }^{4}$ which agglomerates into small metallic clusters after post-growth annealing. ${ }^{5,6} \mathrm{With}$ the formation of arsenic precipitates the electrical and optical properties of LT-GaAs change significantly. ${ }^{3-6}$ For example, annealing at temperatures above $600{ }^{\circ} \mathrm{C}$ usually results in LT-GaAs with extremely high resistivity. However, the mechanism of such high resistivity is still in debate. Some believe that it was the result of depletion of carriers by the Schottky barrier at the surface of the metallic As precipitates, ${ }^{5,6}$ while others suggested a compensation model of defects similar to the conventional semi-insulating (SI) GaAs. ${ }^{4}$ The change of carrier and defect densities inevitably changes the Fermi level, and the determination of which is therefore important in the study of defect-state evolution in post-growth annealing.

Photoreflectance (PR) is extensively used to study the surface electric field and the related Fermi level of semiconductors. However, so far no Franz-Keldysh Oscillation (FKO) produced by the electric field inside LT-GaAs was ever detected except for samples annealed at above $700{ }^{\circ} \mathrm{C}$. $^{7,8} \mathrm{We}$ believe that this is due to the highly nonuniform electric field and the extremely narrow surface depletion region which are the results of high density of defects and traps in LT-GaAs samples. ${ }^{4}$ As a result, the Fermi level of LT-GaAs could only be determined indirectly by PR measurements of the electric field in the adjacent normal GaAs region near a LT-GaAs/ GaAs interface. ${ }^{9}$

Here we report a direct measurement of the surface potential of LT-GaAs by reflectance-difference spectroscopy (RDS). Recent RDS studies of doped GaAs showed strong anisotropy resonances at the critical points $E_{1}$ and $E_{1}+\Delta_{1}$ due to the electro-optic (LEO) effect generated by the sur- face electric field. ${ }^{10-12}$ In this paper we present our RDS study of a series of LT-GaAs samples annealed at different temperatures from 300 to $850{ }^{\circ} \mathrm{C}$. Similar resonances are observed for these samples at the same GaAs critical energies. The line shape of the resonances evidently show that the sign of the surface potential is the same as that of $n$-type conventional GaAs, and the surface space-charge region is indeed very narrow $(\ll 200 \AA)$. This enables us to determine the value of the surface potential without a knowledge of the actual space-charge density, and the effects of post-growth annealing on the Fermi level in LT-GaAs.

The LT-GaAs samples used in this study were grown by a Riber-32p molecular-beam-epitaxy (MBE) system at $250{ }^{\circ} \mathrm{C}$ using a Ga-to-As beam flux ratio of 10 under arsenic-stable growth condition. A 1000- $\AA$ GaAs buffer layer was grown first on (001) liquid-encapsulated Czochralski SI-GaAs substrates at $580{ }^{\circ} \mathrm{C}$. The substrate temperature was then lowered to $250{ }^{\circ} \mathrm{C}$ and a $2-\mu$ m-thick LT-GaAs layer was grown at a growth rate of $1 \mu \mathrm{m}$ per hour. No dopants of any kind were intentionally introduced. After growth, the wafer was cut into pieces. Some of them were annealed at 300, 350, $400,450,500$, and $600{ }^{\circ} \mathrm{C}$ for $30 \mathrm{~min}$ in vacuum or nitrogen environment, while others were subjected to rapid thermal annealing (RTA) at 700,800 , and $850{ }^{\circ} \mathrm{C}$ for $10 \mathrm{~s}$ in arsenic environment. All samples studied were of a high degree of crystalline, as was confirmed by double-crystal x-raydiffraction measurements. Arsenic precipitates were observed in samples annealed at $600{ }^{\circ} \mathrm{C}$ by transmission electron microscopy. In addition, some doped conventional MBE GaAs samples of different carrier densities were grown in a separate system for comparison.

The RDS setup was essentially the same as the one employed earlier. ${ }^{13}$ It was set in a configuration in which only the in-plane anisotropy was measured. Regular photoreflectance was also measured for all the samples. However, only the $n$-type GaAs sample grown by regular MBE showed 


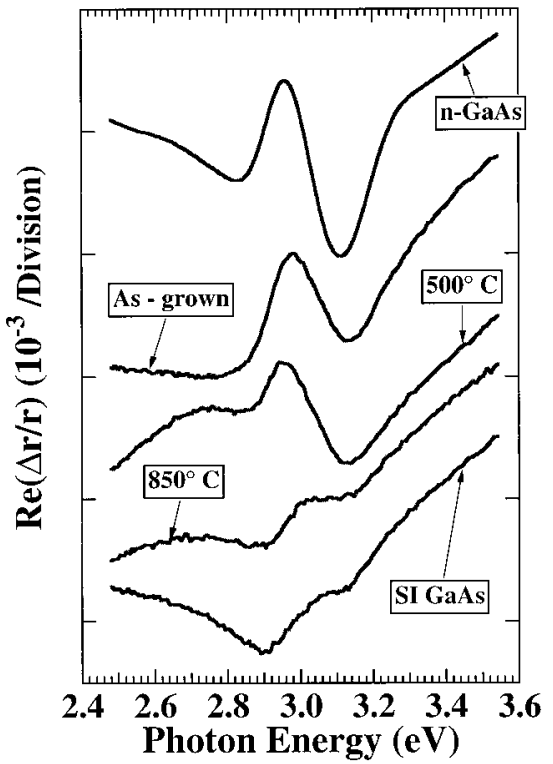

FIG. 1. The real part of the reflectance difference spectra of a semi-insulating GaAs, an $n$-type GaAs, and three LT-GaAs grown on a (001) substrate and annealed at different temperatures.

FKO, which provided another way to determine the surface electric field.

Figure 1 shows the in-plane anisotropy spectra of a semiinsulating (001)-oriented GaAs substrate, an $n$-type GaAs epilayer, and three LT-GaAs samples. The three LT-GaAs samples are the as-grown sample, the sample annealed at $500{ }^{\circ} \mathrm{C}$, and the sample annealed at $850^{\circ} \mathrm{C}$, respectively. The principal axes of the anisotropy are along the [110] and the [110] directions. The optical anisotropy of the SI-GaAs sample is typical and very similar to that found in other SI-GaAs substrates with even unpolished surfaces, and undoped MBE GaAs samples. It was suggested that this optical anisotropy is due to the structure anisotropy of (001) GaAs surface, ${ }^{12}$ even when the surface is covered by an oxide layer. Compared to that of the SI-GaAs substrate, the anisotropy spectra of the $n$-type GaAs shows an additional positive resonance near $E_{1}=2.95 \mathrm{eV}$, and a negative resonance near $E_{1}+\Delta_{1}=3.15 \mathrm{eV}$. This is typical of the $n$-type GaAs samples reported previously, ${ }^{12}$ and is resulted from the LEO effect produced by the surface electric field. ${ }^{10,11}$ The structures in the vicinity of $E_{1}$ and $E_{1}+\Delta_{1}$ for the as-grown LT-GaAs samples are similar to that of the $n$-type GaAs sample. This suggests that they are also from the LEO effect. The amplitude of the LEO structures for the LT-GaAs sample annealed at $500{ }^{\circ} \mathrm{C}$ is reduced as compared to that of the as-grown one, and the LEO structures almost disappear for the samples after $850{ }^{\circ} \mathrm{C}$ RTA. This indicates that the surface electric field of LT-GaAs can be greatly altered by the post-growth annealing. For doped normal GaAs, the surface electric field is determined by the surface potential and the density of the space charges. To find the surface potential, the doping density must be separately measured. However, as the following discussions will show, for LT-GaAs the surface potential can be directly determined from the LEO resonance amplitude without the knowledge of the net space-charge density.

In order to see clearly the difference of the LEO part of

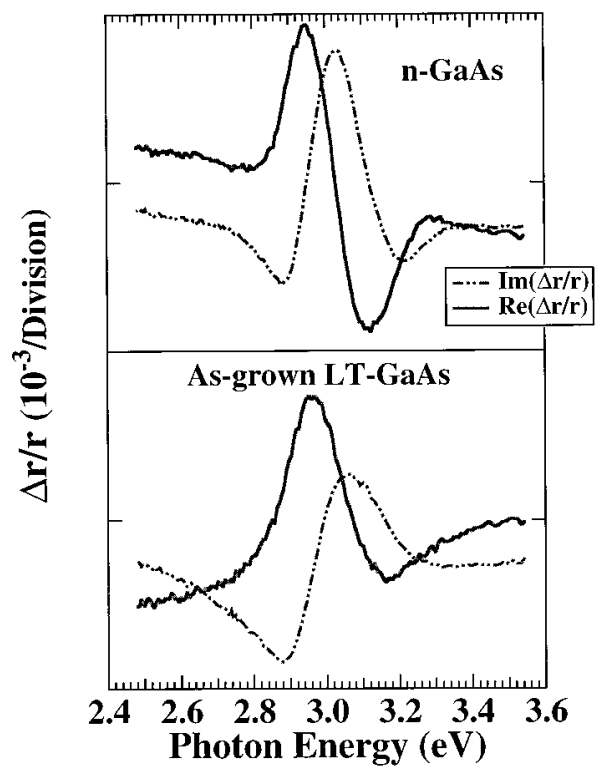

FIG. 2. The linear electro-optic part of the reflectance difference spectra of an $n$-type GaAs and the as-grown LT-GaAs sample.

RDS between the $n$-type GaAs and the LT-GaAs samples, we plot only their LEO component, which are obtained by subtracting the SI-GaAs RDS spectra from their RDS spectra. Typical results are shown in Fig. 2. For the $n$-type GaAs sample, the real part of RDS consists of a positive peak at $E_{1}$ and a negative peak at $E_{1}+\Delta_{1}$, and the imaginary part consists of a positive peak between the $E_{1}$ and $E_{1}+\Delta_{1}$ energies. For LT-GaAs, the real part of its RDS is similar to the imaginary part of the $n$-doped GaAs, while its imaginary part is similar to the negative real part of $n$-doped GaAs. The apparent difference between the two is a factor $-i$ which can be explained as follows. All other LT-GaAs samples show the same resonances but with different amplitudes. The resonance positions of the LT-GaAs are slightly redshifted as compared to that of the $n$-type GaAs. This is due to the lattice expansion by As interstitials.

The LEO part of RDS measures the difference of the dielectric function along the two perpendicular directions in the surface plain. When the electric field varies with the depth, RDS measures an averaged difference of the dielectric function in the two directions over the light penetration length $\langle\Delta \varepsilon\rangle$, which is given by ${ }^{12}$

$$
\langle\Delta \varepsilon\rangle=-2 i k \int_{-d}^{0} e^{-2 i k z} \Delta \varepsilon(z) d z
$$

where $\Delta \varepsilon(z)$ is the LEO-induced difference of the dielectric function proportional to the electric field $F(z), d$ is the space-charge region depth, and $k$ is the complex wave vector of light. Because $\Delta \varepsilon(z)$ is zero outside the space-charge region, and the exponential term $\exp (-2 i k z)$ approaches zero beyond the penetration depth, it is apparent that $\langle\Delta \varepsilon\rangle$ depends on the magnitude of the penetration depth or the depth of the space-charge region, whichever is smaller. For a photon energy of $3 \mathrm{eV}$, the penetration depth is about $170 \AA$ for GaAs. For our $n$-type GaAs sample, the depth of the spacecharge region is about $1500 \AA$, with the density of space 


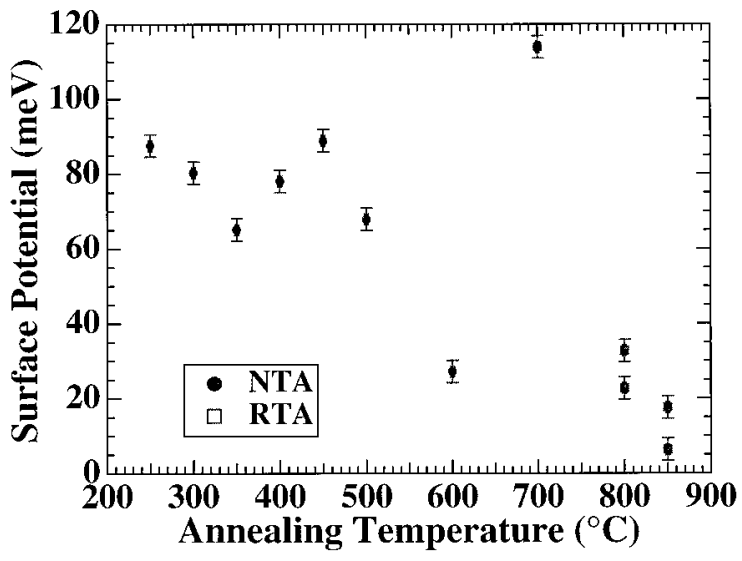

FIG. 3. The surface potential of LT-GaAs as a function of postgrowth annealing temperatures. The closed points are for thermal annealing, while the open squares are for rapid thermal annealing.

charges in the range of $10^{17} \mathrm{~cm}^{-3}$ and the surface potential $V_{b}=0.7 \mathrm{eV}$, as separately measured from the PR spectrum and the Hall data. In this case $\Delta \varepsilon(z)$ can be approximated by $\Delta \varepsilon(0)$ in Eq. (1), which leaves

$$
\langle\Delta \varepsilon\rangle \cong \Delta \epsilon(0)=\beta F_{s},
$$

where $F_{s}$ is the surface electric field, and $\beta$ is the LEO coefficient.

For the LT-GaAs the surface potential is about $0.1 \mathrm{eV}$, and the space-charge density is about $10^{20} \mathrm{~cm}^{-3}$. This produces a narrow space-charge region with a depth on the order of $10 \AA$, much smaller than the light penetration depth. Under this condition, the exponential term in the integral of Eq. (1) can be neglected. Equation (1) is then converted into an integral of the electric field, since $\Delta \varepsilon(z)$ is proportional to the electric field at $z$ based on the LEO effect. The final result can be simplified as

$$
\langle\Delta \varepsilon\rangle=-2 i k \beta V_{b} .
$$

It is clear that the different line shape of the LEO structures of the $n$-type GaAs and the LT-GaAs samples is simply due to the factor $-i$. This will change the imaginary (real) part of the RDS for the $n$-type GaAs to the real (negative imaginary) part for the LT-GaAs, as is clearly shown in Fig. 2, since the wave vector $k$ is mostly real. Moreover, Eq. (3) shows that the LEO resonance of LT-GaAs is determined only by the surface potential $V_{b}$, and is independent of the space-charge density.

The LEO coefficient $\beta$ of (100) GaAs was obtained by comparing the surface field obtained from the PR data and the RDS peak-to-peak amplitude of the $n$-type sample at the $E_{1}$ and $E_{1}+\Delta_{1}$ energies. Our results are consistent with those reported in Ref. 12. Assuming that the LEO coefficient of the LT-GaAs is the same as normal GaAs, the effective surface electric field of the LT-GaAs samples, $2 k V_{b}$, can then be obtained. Finally the surface potential is calculated according to Eq. (3). Figure 3 shows the surface potential versus the annealing temperature. It is clear that the surface potential depends strongly on the annealing temperature. The surface potential decreases from $89 \mathrm{mV}$ in the as-grown sample, to about $67 \mathrm{mV}$ in the sample annealed at $350{ }^{\circ} \mathrm{C}$.
For higher annealing temperatures it begins to increase, and reaches a maximum value of $90 \mathrm{mV}$ at $450{ }^{\circ} \mathrm{C}$, and then drops down to about $30 \mathrm{mV}$ for the sample annealed at $600{ }^{\circ} \mathrm{C}$. RTA at $850^{\circ} \mathrm{C}$ further reduces the potential to less than $10 \mathrm{mV}$. It is clear that the Fermi level is not yet pinned at $600{ }^{\circ} \mathrm{C}$. These results are consistent with the earlier work by Look et al. ${ }^{4}$ Furthermore, RTA at $700{ }^{\circ} \mathrm{C}$ increases the surface potential at $115 \mathrm{meV}$.

Assuming that post-growth annealing does not affect the Fermi level at the surface, the change of the surface potential would then result from the shift of the bulk Fermi level in the forbidden gap of LT-GaAs. The nonmonotonic change of the Fermi level with the annealing temperature indicates that there are competing mechanisms during the annealing process, and a simple model of Fermi-level pinning to As precipitates is not adequate. It is well known that most of the excess As in LT-GaAs is in the form of defects such as As interstitials $\left(\mathrm{As}_{i}\right)$, As antisites $\left(\mathrm{Aa}_{\mathrm{Ga}}\right)$, Ga vacancies $\left(V_{\mathrm{Ga}}\right)$, or small complexes of these defects. $\mathrm{As}_{\mathrm{Ga}}$ serves as a deep donor near the midgap, $V_{\mathrm{Ga}}$ is believed to be a deep acceptor at $0.28 \mathrm{eV}$ above the valence band, ${ }^{14}$ while $\mathrm{As}_{i}$ may not be electrically active but expands the lattice of LT-GaAs. The Fermi level is usually several $k T$ above the position of $\mathrm{As}_{\mathrm{Ga}}$ depending on the ratio of the density of $\mathrm{As}_{\mathrm{Ga}}$ to the net density of acceptors below the midgap. When the ratio decreases, more $\mathrm{As}_{\mathrm{Ga}}$ are ionized, leaving a lower Fermi level and a lower surface potential. During the annealing at 300$350{ }^{\circ} \mathrm{C}$, only $\mathrm{As}_{i}$ is mobile. ${ }^{15}$ It moves to form As precipitates and release the strain. The processes $\left(\mathrm{As}_{\mathrm{Ga}} \rightarrow \mathrm{As}_{i}\right.$ $\left.+V_{\mathrm{Ga}}\right),\left(\mathrm{As}_{i}-V_{\mathrm{Ga}}\right.$ complex $\left.\rightarrow \mathrm{As}_{i}+V_{\mathrm{Ga}}\right)$ and $\left(\mathrm{As}_{i}-\mathrm{As}_{\mathrm{Ga}}\right.$ complex $\rightarrow \mathrm{As}_{i}+\mathrm{As}_{\mathrm{Ga}}$ ) take place to balance the decrease of $\mathrm{As}_{i}$. These processes will change the $\mathrm{As}_{\mathrm{Ga}}$ concentration slightly, while increasing that of $V_{\mathrm{Ga}}$, thus increasing the $V_{\mathrm{Ga}} / \mathrm{As}_{\mathrm{Ga}}$ ratio. As a result, the Fermi level moves away from the conduction band, and leaves a lower surface potential. At temperatures of $400-450{ }^{\circ} \mathrm{C}, V_{\mathrm{Ga}}$ becomes mobil. There are several possibilities for it. The first is that $V_{\mathrm{Ga}}$ assists the diffuse of $\mathrm{As}_{\mathrm{Ga}}$ to form As precipitates and finally being absorbed by the $\mathrm{Ga}$ atoms expelled by the precipitates. ${ }^{16}$ The second possibility is that $V_{\mathrm{Ga}}$ collides with As, and forms the complex $V_{\mathrm{As}}-\mathrm{As}_{\mathrm{Ga}}$, which is probably a donor of $0.17 \mathrm{eV}$ below the conduction band. ${ }^{14}$ The third is just to form vacancy clusters. ${ }^{17}$ These processes will all cause the $V_{\mathrm{Ga}}$ density to decrease, which causes the Fermi level to shift back to the conduction band, and increases the height of the surface potential. When the annealing temperature is higher than $500{ }^{\circ} \mathrm{C}$, most of $\mathrm{As}_{\mathrm{Ga}}$ moves to form the As precipitates, which moves the Fermi level to the midgap again. The change of the surface potential therefore comes from the increase and decrease of $V_{\mathrm{Ga}}$ and $\mathrm{As}_{\mathrm{Ga}}$ at different annealing temperatures. It was observed previously that the ionized $\mathrm{As}_{\mathrm{Ga}}$ would increase from $4 \times 10^{18}$ to $1 \times 10^{19} \mathrm{~cm}^{-3}$ after annealing at $350{ }^{\circ} \mathrm{C}$, and then decreases to $1 \times 10^{18} \mathrm{~cm}^{-3}$ after $400{ }^{\circ} \mathrm{C}$ annealing. ${ }^{4}$ Noting that the density of the ionized $\mathrm{As}_{\mathrm{Ga}}$ is approximately equal to that of $V_{\mathrm{Ga}}$, this result supports our discussion above. The same discussions also apply to the RTA samples. The relative variation of the deep donors and acceptor usually shift the Fermi level by several $k T$ which is consistent with the data in Fig. 3 that show a maximum shift of about $100 \mathrm{mV}$.

In summary, we have shown that RDS is an effective tool 
for the study of surface potential of LT-GaAs, and that the surface electric field of LT-GaAs is limited to an extremely narrow space-charge region. Unlike the case of doped normal GaAs, the amplitude of the LEO resonances of LT-GaAs is determined by the surface potential instead of the surface electric field. The change of the surface potential, and therefore the Fermi level, can be determined to a precision of better than $5 \mathrm{meV}$. Such precision is very difficult to achieve in conventional PR measurements of the electric field at LT$\mathrm{GaAs} / \mathrm{GaAs}$ interfaces, since the potential involved is of the order of $0.5 \mathrm{eV}$. The effect of the post-growth annealing on the surface potential of LT-GaAs shows a complicated movement of the Fermi level in LT-GaAs. The evolution of the Fermi level as a function of annealing temperature shows that the Fermi level is not determined by pinning to As precipitates alone. At low annealing temperatures it is determined by a process of various defect formation and annihilation, while at high annealing temperatures the effect of surface states at the As precipitates may also be taken into account. The Fermi level is not pinned to the surface state of As precipitates which are present after annealing at $600{ }^{\circ} \mathrm{C}$ and above, but still moves at higher annealing temperatures.

This work was supported by the Research Grant Council Grant 609/95P from the Hong Kong Government, and most of the experiments were performed in the William Mong's Semiconductor Cluster Laboratory at Hong Kong University of Science and Technology.

${ }^{8}$ T. M. Cheng, C. Y. Chang, T. M. Hsu, W. C. Lee, and J. H. Huang, J. Appl. Phys. 77, 2124 (1995).

${ }^{9}$ H. Shen, F. C. Rong, R. Lux, J. Pamulapati, M. Taysing-Lara, M. Dutta, E. H. Poidexter, L. Calderon, and Y. Lu, Appl. Phys. Lett. 61, 1585 (1992).

${ }^{10}$ S. E. Acosta-Ortiz and A. Lastras-Martinez, Phys. Rev. B 40, 1426 (1989).

${ }^{11}$ S. E. Acosta-Ortiz, J. Appl. Phys. 70, 3239 (1991).

${ }^{12}$ H. Tanaka, E. Colas, I. Kamiya, D. E. Aspnes, and R. Bhat, Appl. Phys. Lett. 59, 3443 (1991).

${ }^{13}$ Z. Yang, I. K. Sou, Y. H. Yeung, G. K. L. Wong, Jie Wang, Cai-xia Jin, and Xiao-Yuan Hou, J. Vac. Sci. Technol. B 14, 2973 (1996).

${ }^{14}$ Z.-Q. Fang and D. C. Look, Appl. Phys. Lett. 63, 219 (1993).

${ }^{15}$ M. Uematsu, P. Werner, M. Schultz, T. Y. Yan, and U. Gosele, Appl. Phys. Lett. 67, 2863 (1995); Masashi Uematsu, Peter Werner, Matthias Schultz, The Y. Tan, and Ulrich M. Gosele, Appl. Phys. Lett. 67, 2863 (1995).

${ }^{16}$ D. E. Bliss, W. Walukiewicz, J. W. Ager III, E. E. Haller, K. T. Chan, and S. Tanigawa, J. Appl. Phys. 71, 1699 (1992).

${ }^{17}$ J. Stormer, W. Triftschauser, N. Hozhabri, and K. Alavi, Appl. Phys. Lett. 69, 1867 (1996). 